\title{
Short communication: Association of disease incidence and adaptive immune response in Holstein dairy cows
}

\author{
K. A. Thompson-Crispi, ${ }^{\star 1}$ B. Hine, $†$ M. Quinton,‡ F. Miglior,§\# and B. A. Mallard ${ }^{\star}$ \\ *Department of Pathobiology, University of Guelph, Guelph, Ontario, N1G 2W1, Canada \\ †Dairy Science and Technology, AgResearch Limited, Hamilton, New Zealand, 3240 \\ †Department of Animal and Poultry Science, University of Guelph, Guelph, Ontario, N1G 2W1, Canada \\ §Guelph Food Research Center, Agriculture and Agri-Food Canada, Guelph, Ontario, Canada, N1G 5C9 \\ \#Canadian Dairy Network, Guelph, Ontario, N1K 1E5, Canada
}

\section{ABSTRACT}

The objective of this study was to use previously calculated estimated breeding values for cell- (CMIR) and antibody-mediated immune responses (AMIR) to determine associations between immune response (IR) and economically important diseases of dairy cattle. In total, 699 Holsteins were classified as high, average, or low for CMIR, AMIR, and overall IR (combined CMIR and AMIR), and associations with mastitis, metritis, ketosis, displaced abomasums, and retained fetal membranes were determined. The incidence of mastitis was higher among average cows as compared with cows classified as high AMIR [odds ratio $(\mathrm{OR})=2.5$ ], high CMIR (OR = 1.8), or high IR $(\mathrm{OR}=1.8)$. Low-CMIR cows had a higher incidence of metritis $(\mathrm{OR}=11.3)$ and low-IR cows had a higher incidence of displaced abomasum $(\mathrm{OR}=4.1)$ and retained fetal membrane $(\mathrm{OR}=2.8)$ than did average responders. Results of this study show that cows classified as high immune responders have lower occurrence of disease, suggesting that breeding cattle for enhanced IR may be a feasible approach to decrease the incidence of infectious and metabolic diseases in the dairy industry.

Key words: immune response, disease, health, genetics

\section{Short Communication}

Selection for enhanced immune response (IR) is expected to decrease the incidence of disease and improve health in dairy cattle (Abdel-Azim et al., 2005; Mallard et al., 2011). The use of antibody-mediated (AMIR) and cell-mediated IR (CMIR) as indicator traits of adaptive IR in cattle and other species has proved useful in identifying individuals with high or low IR profiles (Mallard et al., 1992; Sarker et al., 2000;

Received November 28, 2011.

Accepted February 27, 2012.

${ }^{1}$ Corresponding author: kthomp02@uoguelph.ca
Heriazon et al., 2011). In a previous study that evaluated antibody responses in Canadian Holsteins it was found that in 2 out of 3 herds tested, cows with a highAMIR phenotype had a lower occurrence of mastitis (Wagter et al., 2000). In a more recent study aimed at comparing regional differences in immune responsiveness of dairy cattle, cows in Alberta were found to have higher AMIR and CMIR than cows in other regions of Canada, which was associated with a lower incidence rate of clinical mastitis (Thompson-Crispi and Mallard, 2012).

Adaptive IR can be broadly categorized into AMIR and CMIR based on function and the types of cytokines and antibody isotypes produced. Antibody-mediated IR generally predominates in host defense against extracellular pathogens and is characterized by production of the cytokines IL- 4 and IL-13 and antibody of the IgG1 isotype. On the other hand, CMIR predominates against intracellular pathogens, and can be characterized by production of IFN $\gamma$ and antibody of the IgG2 isotype (Estes and Brown, 2002; Zhu and Paul, 2008). Antibody-mediated IR and CMIR have been shown to be negatively genetically correlated in dairy cattle, although it is accepted that both responses work in coordination for optimal host protection (Hernández et al., 2006; Hine et al., 2011; Thompson-Crispi et al., 2012). The negative genetic correlation that exists between AMIR and CMIR is important when considering breeding strategies for disease resistance. Breeding for resistance to a particular disease has the potential to leave individuals susceptible to other diseases. Because AMIR and CMIR predominate in control of different types of pathogens, an optimal balance between both are required for broad-based disease resistance to many disease-causing organisms. Further, diseases such as mastitis (the inflammation of the mammary gland) can be caused by a multitude of organisms that use diverse mechanisms to infect and persist in the host. For example, Staphylococcus aureus, a common mastitis pathogen, is an extracellular organism that has been shown to survive intracellularly as small colony 
variants (Atalla et al., 2010a). These small colony variants have the ability to evade the immune system, and have also been shown to induce CMIR (Atalla et al., 2010b). Therefore, cows require an optimal balance of both AMIR and CMIR for protection against mastitiscausing pathogens.

Other economically important diseases, such as metritis and retained fetal membranes (RFM) have been shown to have innate immune involvement, mainly through the action of neutrophils. Metritis is the inflammation of the uterus resulting from bacterial infection after calving and requires prompt neutrophil responses for clearance of bacteria (Galvão et al., 2011). Retained fetal membranes also involve the innate immune system, as normal placenta expulsion requires increase in leukocyte chemotaxis, with IL-8 playing an important role in neutrophil recruitment to the cotyledon (Beagley et al., 2010). Appropriate innate IR are critical in initiating and regulating the adaptive responses such as AMIR and CMIR. Other diseases such as ketosis and displaced abomasums (DA) may not have direct immune involvement but can be indirectly affected by compromised IR that occurs around calving (LeBlanc, 2010).

The objectives of this study were 1) to use standardized IR breeding values to categorize 699 commercial cows as high, average, or low immune responders for AMIR, CMIR, and overall IR; and 2) to correlate IR with the incidence of economically important diseases of dairy cattle including mastitis, metritis, RFM, ketosis, and DA. Results of this study further demonstrate the benefits of identifying high immune responders. Previously, selection for high IR in pigs resulted in improved IR, better response to vaccination, and higher weight gain. (Mallard et al., 1992; Wilkie and Mallard, 1999). The current study suggests that breeding dairy cattle for enhanced immune responsiveness may be associated with improved overall disease resistance.

A total of 875 commercial Holstein cows from a large herd in north central Florida were enrolled for IR testing as described previously by Heriazon (2007). Cows confirmed pregnant and in good health, were enrolled in the study $8 \mathrm{wk}$ before their expected calving date. Immune response traits (CMIR and AMIR) were evaluated as described previously (Wagter et al., 2000; Hernández et al., 2005). Briefly, cows were immunized with known type- 1 and type-2 test antigens (US Patent \#7,258,858; Wagter and Mallard, 2007) to stimulate adaptive IR at wk $-8,-3$, and 0 relative to calving. Blood samples were then collected at wk $-8,-3,0$, and +2 relative to calving and serum IgG antibody to the type- 2 test antigen determined by ELISA as an indicator of AMIR. Week -8 values were subtracted from wk $-3,0$, and +2 . The CMIR was evaluated using a delayed-type hypersensitivity skin test to the type-1 test antigen assessed $1 \mathrm{wk}$ after calving. Pedigree information was available for 871 cows, giving a total of 1,536 records (performed by the Canadian Dairy Network, Guelph, ON, Canada). Genetic parameters for each trait were estimated as described previously (Heriazon, 2007). Briefly, a 4-trait animal model was used to estimate genetic and residual error variance and covariance parameters using variance component estimation software (VCE5; Kovac et al., 2002). The traits used in the model were CMIR at wk +1 and AMIR assessed at $\mathrm{w}-3,0$, and +2 relative to calving. Parameter estimation software (PEST; Parameter Estimation Inc., Sandy, UT) was used to estimate breeding values for the 4 traits. The EBV for AMIR used to determine associations with disease incidence was calculated by averaging the EBV for AMIR at each of the 3 time points. For use in this study, EBV for each trait were standardized to have a mean of 0 and standard deviation of 1 . Standardized EBV were then used to classify cows as high, average, or low immune responders for AMIR, CMIR, and overall IR. The EBV for overall IR was calculated by averaging the EBV for AMIR and CMIR. Cows with an EBV $>+1$ or $<-1$ standard deviation for each trait were considered high or low responders, respectively.

Diseases assessed in the study included mastitis, metritis, RFM, ketosis, and DA. Disease incidence data were recorded as described previously by DeLapaz (2008), with incidence of disease recorded as a binary trait during the lactation immediately following IR testing. Disease recording was performed by trained personnel blinded to IR classification. Mastitis was recorded to 365 DIM as light, medium, or severe. Severity was considered light if milk was slightly watery with minimal clots and no systemic signs were present, medium if milk had a substantial number of clots but no systemic signs, and severe if the milk was watery with a substantial number of clots and systemic signs of infection were evident. Metritis was recorded to 30 DIM and was considered light if abnormal vaginal discharge and a palpable uterine lumen were present; medium if purulent vaginal discharge with enlarged, not-flaccid uterus was present; and severe if purulent foul-smelling vaginal discharge with an enlarged uterus was present. Ketosis was also recorded to 30 DIM and was considered light if urine contained 15 to $39 \mathrm{mg}$ of ketone bodies/dL, medium if 40 to $79 \mathrm{mg} / \mathrm{dL}$, and severe if $>80$ $\mathrm{mg} / \mathrm{dL}$. The incidence of DA was recorded to $50 \mathrm{DIM}$ and RFM were defined as placental retention $24 \mathrm{~h}$ postpartum (DeLapaz, 2008). Complete IR testing results, $\mathrm{EBV}$, and disease records were available for 699 cows. Disease incidence by IR category was analyzed with a linear mixed model using PROC MIXED (SAS version 
Table 1. Incidence of disease in low, average (Ave), and high immune responders within each of the immune response (IR) categories [antibodymediated IR (AMIR), cell-mediated IR (CMIR), and overall IR (IR)] based on standardized EBV ${ }^{1}$

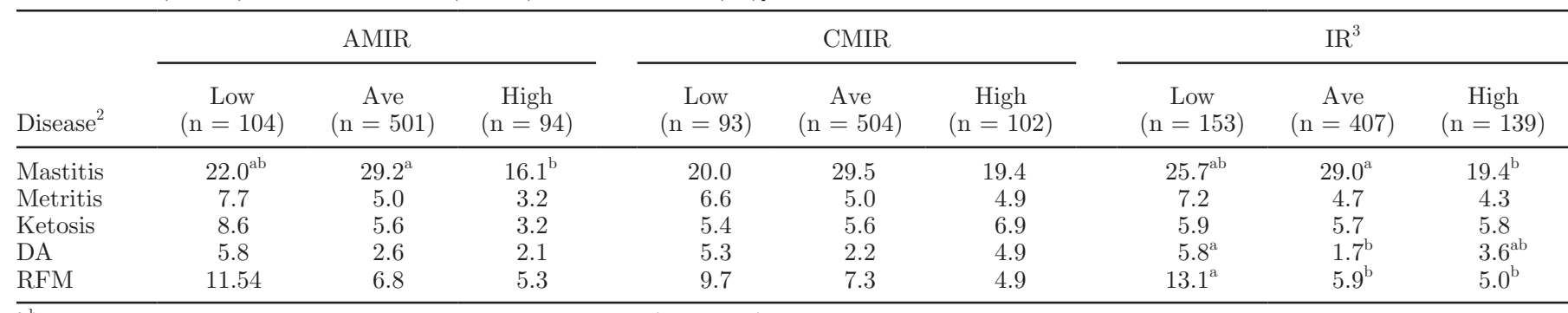

a,b Incidence within a row with different superscripts differ $(P<0.05)$, within IR category only.

${ }^{1}$ For each trait, EBV were standardized to have a mean of 0 and a standard deviation of 1 . Cows with a standardized EBV of $<-1$, between -1 and +1 , and $>+1$ were considered low, Ave, and high responders, respectively.

${ }^{2} \mathrm{DA}=$ displaced abomasums; $\mathrm{RFM}=$ retained fetal membranes.

${ }^{3}$ The EBV for overall immune response (IR) was calculated as the mean of standardized EBV for AMIR and CMIR.

9.1.3; SAS Institute Inc., Cary, NC). Contrasts between high, average, and low immune responders within each IR trait were evaluated using Tukey test statistics. To estimate the odds ratio of disease, a logistic regression was performed using the PROC LOGISTIC procedure (SAS version 9.1.3).

Mastitis was the most frequently occurring disease with $23.6 \%$ of cows experiencing at least 1 case within the lactation immediately following IR testing, followed by $\operatorname{RFM}(7.3 \%)$, metritis $(6.9 \%)$, ketosis $(5.7 \%)$, and DA $(3.0 \%)$. These results are consistent with previous estimates of disease incidence during lactation in Holsteins (Kelton et al., 1998; Ghavi Hossein-Zadeh and Ardalan, 2011). Comparisons of disease incidence between high, average, and low immune responders for each IR trait are presented in Table 1 and odds ratios describing the likelihood of disease occurrence between groups are presented in Table 2 .

The incidence of mastitis in high-AMIR cows was $16.1 \%$, which was significantly lower than those classified as average (29.2\%). The incidence of mastitis among low-AMIR cows $(22.0 \%)$ was not statistically different than cows classified as high or average for AMIR (Table 1). Average-AMIR cows were 2.5 times more likely to have a case of severe mastitis than were high-AMIR cows (Table 2), indicating that cows with enhanced an-

Table 2. Comparison of likelihood of disease as represented by the odds ratio of disease among low, average (Ave), and high immune responders within each of the immune response (IR) categories [antibody-mediated IR (AMIR), cell-mediated IR (CMIR), and overall IR (IR)] based on standardized EBV ${ }^{1}$

\begin{tabular}{lllll}
\hline $\begin{array}{l}\text { IR } \\
\text { category }\end{array}$ & Disease $^{2}$ & $\begin{array}{l}\text { Groups } \\
\text { compared }\end{array}$ & $\begin{array}{c}\text { Odds } \\
\text { ratio }\end{array}$ & $\begin{array}{c}\text { Wald } \\
95 \% \text { CI }\end{array}$ \\
\hline AMIR & & & & \\
& S mastitis & Ave vs. high & 2.486 & $1.043-5.922$ \\
& M or S mastitis & Ave vs. high & 2.198 & $1.151-4.197$ \\
& L, M, or S mastitis & Ave vs. high & 2.158 & $1.153-4.039$ \\
CMIR & M or S mastitis & Ave vs. low & 1.792 & $1.006-3.193$ \\
& M or S mastitis & Ave vs. high & 1.805 & $1.013-3.214$ \\
IR & Low vs. Ave & 11.258 & $1.010-125.479$ \\
& M mastitis & Ave vs. high & 1.783 & $1.056-3.010$ \\
& M or S mastitis & Ave vs. high & 1.709 & $1.020-2.862$ \\
& L, M, or S mastitis & Ave vs. high & 1.688 & $1.024-2.782$ \\
& DA (16 DIM) & Low vs. Ave & 4.112 & $1.144-14.778$ \\
& DA (50 DIM) & Low vs. Ave & 3.571 & $1.306-9.766$ \\
& RFM & Low vs. high & 2.836 & $1.160-6.932$ \\
& RFM & Low vs. Ave & 2.400 & $1.284-4.485$ \\
\hline
\end{tabular}

${ }^{1}$ For each trait, EBV were standardized to have a mean of 0 and a standard deviation of 1 . Cows with a standardized EBV of $<-1$, between -1 and +1 , and $>+1$ were considered low, Ave, and high responders, respectively.

${ }^{2}$ Only diseases that had significant odds ratios are presented. Severity of mastitis was scored as light (L), medium $(\mathrm{M})$, or severe $(\mathrm{S})$. Incidences of mastitis and metritis were recorded to 365 and $30 \mathrm{DIM}$, respectively. DA $=$ displaced abomasums; RFM $=$ retained fetal membranes 
tibody responses are better immunologically equipped to respond to the persistent environmental challenge posed by mastitis-causing pathogens. Mastitis pathogens tend to be extracellular in nature, requiring type 2 , or more general, antibody responses, to protect the host and, therefore, high antibody responses could be expected to provide enhanced protection, as found in this study. High-AMIR cows also had a lower incidence of metritis, ketosis, DA, and RFM compared with lowAMIR cows (Table 1); however, these differences were not statistically significant possibly due to the small sample size and low incidence rate.

Associations between CMIR and incidence of disease were also investigated. Although not significant, highCMIR cows had fewer incidences of mastitis, metritis, DA, and RFM than did low-CMIR cows (Table 1). The incidence of mastitis was $19.4 \%$ in high-CMIR cows, $29.5 \%$ in average-, and $20.0 \%$ in low-CMIR cows. This correlated with average-CMIR cows being 1.8 times more likely to develop medium or severe mastitis than high-CMIR cows (Table 2). It would be expected that low immune-responding cows would have the highest incidence of mastitis as was seen with the other diseases. However, average responders, based on all IR categories, had the highest incidence of mastitis (but not significantly different than low responders) and this may be due to the standard deviation classification method causing a larger number of cows to be in the average group. Nonetheless, previous studies by our group when taken together find the highest disease occurrence (including mastitis) in low-IR cows, compared with average or high responders. Low-CMIR cows were 11.3 times more likely to develop metritis by 30 DIM than were average-CMIR cows.

Exposure of the uterus to bacteria is common after calving, and successful clearance of bacteria requires a prompt neutrophil response. Neutrophil recruitment is initiated by a proinflammatory cytokine milieu created in the endometrium after local recognition of bacteria through toll-like receptors. Low-level production of proinflammatory cytokines, including tumor necrosis factor $\alpha(\mathrm{TNF} \alpha)$, IL-1 $\beta$, and IL-6, in the endometrium of cows soon after calving has been associated with the development of metritis (Galvão et al., 2011). The CMIR test used in this study gives a general indication of the ability of cows to mount cellular responses, and has been associated with increased cell infiltration at the test site, including neutrophils (Hernández et al., 2005). Interestingly, in a study that measured gene expression in high- and low-AMIR and -CMIR cows it was found that low-CMIR cows had lower transcriptional activity for the neutrophil chemoattractant IL-8 (NinoSoto et al., 2008). Additionally, an effective CMIR is often required to control infections that are chronic in nature. Although not tested here, a decreased influx of neutrophils in the endometrium at calving may be associated with lower overall ability for cows to initiate CMIR, as low-CMIR cows were also more likely to develop metritis. Taken together, this may suggest that using CMIR as an indicator trait of adaptive immunity may also identify cows with a better ability to initiate responses necessary in the control of diseases such as metritis.

Cows categorized as high for overall IR had fewer incidences of mastitis, DA, and RFM than did low-IR cows. The incidence of mastitis in high-IR cows was $19.4 \%$, which was significantly lower than in average-IR cows with $29.0 \%$, and lower (though not significant) than low-IR cows with $25.7 \%$ (Table 1). Average-IR cows were 1.8 times more likely to develop a case of mastitis by 365 DIM as compared with the high-IR cows (Table 2). These results suggest that breeding dairy cattle for enhanced overall IR may decrease the incidence and severity of mastitis.

In this study, mastitis was recorded as a binary trait and, therefore, data on persistent or recurring cases was not assessed. This limitation in the disease data may have contributed to the lack of association between low immune-responding cows and higher incidences of mastitis as hypothesized in the study. Cows classified as high IR also had significantly fewer RFM as compared with low-IR cows, with high-IR cows having a 5.0\%, average-IR cows a $5.9 \%$, and low-IR cows a $13.1 \%$ incidence of RFM. Low-IR cows were 2.8 times more likely than high-IR cows and 2.4 times more likely than average-IR cows to have RFM (Table 2). Retained fetal membranes affect reproductive performance and are associated with an increased risk for metritis, ketosis, and mastitis (Beagley et al., 2010; Ghavi Hossein-Zadeh and Ardalan, 2011). Activation of the maternal IR is fundamental in facilitating the breakdown of the placenta after calving. Immunosuppression, common around the time of calving, is known to be a risk factor for RFM, and although not fully understood, this increased risk is associated with decreased leukocyte chemotaxis and phagocytic activity before parturition, and lower levels of IL-8 production, suggesting decreased neutrophil activity (Beagley et al., 2010; Benedictus et al., 2011).

Although, again, not tested here, the finding that cows with lower overall immune responsiveness are at an increased risk for developing RFM emphasizes the fact that adaptive response indicator traits (AMIR and CMIR) are linked with innate responses and that these may be required for normal expulsion of the placenta. Results suggest that breeding for overall enhanced adaptive immune responsiveness may decrease the incidence of diseases such as RFM that put cows at an increased risk for developing mastitis and metritis and 
can decrease production and reproductive performance. Low-IR cows were also at an increased risk of developing DA. The incidence of DA during early lactation was $5.8 \%$ in low-IR cows, which was significantly higher than in average- $(1.7 \%)$ and high- $(3.6 \%)$ IR cows (Table 1). In the period to 16 DIM, low-IR cows were 4.1 times more likely than average-IR cows to have DA (Table 2). High levels of circulating NEFA $(>0.4 \mathrm{mmol} / \mathrm{L})$ in the week before calving are associated with an increased risk of developing DA and RFM (LeBlanc et al., 2005; LeBlanc, 2010). The peripartum period is characterized by an increase in energy requirements for lactation, often resulting in a negative energy balance. The mobilization of fat and release of NEFA at this time have been associated with reduced function of bovine immune cells (Ster et al., 2012) and is a major factor that may contribute to the development of metabolic and infectious diseases (Sordillo et al., 2009; LeBlanc, 2010). Based on results of this study, which suggest that cows with enhanced adaptive IR have a lower incidence of metabolic disease during the peripartum period, it would be of interest to investigate associations between immune responsiveness and negative energy balance associated with lower levels of circulating NEFA around the time of calving. Breeding dairy cattle for enhanced adaptive IR may prove valuable for selecting cows better able to handle increases in energy requirement after calving, thereby decreasing the incidence of metabolic diseases.

Overall, the results of this study show that dairy cattle identified as high immune responders are at a decreased risk of developing diseases including mastitis, metritis, DA, and retained placenta, suggesting that breeding for enhanced overall immune responsiveness will decrease the incidence and severity of infectious and metabolic diseases in dairy cattle. Future studies aimed at investigating associations between innate immune responsiveness and the adaptive IR may provide further explanation of the beneficial associations found in this study of AMIR and CMIR with diseases generally controlled by innate IR.

\section{ACKNOWLEDGMENTS}

This research was supported by grants to B. A. Mallard from the Natural Sciences and Engineering Research Council of Canada (Ottawa, ON, Canada). The authors acknowledge Armando Heriazon (Novartis Animal Health, Prince Edward Island, Canada) and Jason De La Paz (University of Florida, Gainesville) for their help in collecting the immune response and disease data. We thank 2 anonymous reviewers for their helpful suggestions and comments.

\section{REFERENCES}

Abdel-Azim, G. A., A. E. Freeman, M. E. Kehrli Jr., S. C. Kelm, J. L. Burton, A. L. Kuck, and S. Schnell. 2005. Genetic basis and risk factors for infectious and noninfectious diseases in US Holsteins. I. Estimation of genetic parameters for single diseases and general health. J. Dairy Sci. 88:1199-1207.

Atalla, H., C. Gyles, and B. Mallard. 2010a. Persistence of a Staphylococcus aureus small colony variants (S. aureus SCV) within bovine mammary epithelial cells. Vet. Microbiol. 143:319-328.

Atalla, H., B. Wilkie, C. Gyles, K. Leslie, L. Mutharia, and B. Mallard. 2010b. Antibody and cell-mediated immune responses to Staphylococcus aureus small colony variants and their parental strains associated with bovine mastitis. Dev. Comp. Immunol. 34:1283-1290.

Beagley, J. C., K. J. Whitman, K. E. Baptiste, and J. Scherzer. 2010. Physiology and treatment of retained fetal membranes in cattle. J. Vet. Intern. Med. 24:261-268.

Benedictus, L., R. Jorritsma, H. M. Knijn, P. L. Vos, and A. P. Koets 2011. Chemotactic activity of cotyledons for mononuclear leukocytes related to occurrence of retained placenta in dexamethasone induced parturition in cattle. Theriogenology 76:802-809.

De La Paz, J. M. 2008. Using antibody and cell-mediated immune response to test antigens in periparturient dairy cows as a measure of disease resistance. MSc Thesis. University of Florida, Gainesville. Accessed Sep. 19, 2011. http://etd.fcla.edu/UF/UFE0022360/ delapaz_j.pdf.

Estes, D. M., and W. C. Brown. 2002. Type 1 and type 2 responses in regulation of $\mathrm{Ig}$ isotype expression in cattle. Vet. Immunol. Immunopathol. 90:1-10.

Galvão, K. N., N. R. Santos, J. S. Galvão, and R. O. Gilbert. 2011. Association between endometritis and endometrial cytokine expression in postpartum Holstein cows. Theriogenology 76:290-299.

Ghavi Hossein-Zadeh, N., and M. Ardalan. 2011. Cow-specific risk factors for retained placenta, metritis and clinical mastitis in Holstein cows. Vet. Res. Commun. 35:345-354.

Heriazon, A. 2007. Phenotypic and genetic parameters of acquired immune responses to improve dairy cattle health. $\mathrm{PhD}$ Thesis. University of Guelph, Guelph, ON, Canada.

Heriazon, A., K. Hamilton, J. Huffman, B. N. Wilkie, W. Sears, M. Quinton, and B. A. Mallard. 2011. Immunoglobulin isotypes of lactating Holstein cows classified as high, average, and low type-1 or -2 immune responders. Vet. Immunol. Immunopathol. 144:259269.

Hernández, A., M. Quinton, F. Miglior, and B. A. Mallard. 2006. Genetic parameters of dairy cattle immune response traits. Pages 15-18 in Proc. 8th World Congr. Genet. Appl. Livest. Prod., Belo Horizonte, Minas Gerais, Brazil. WCGALP, Belo Horizonte, MG, Brazil.

Hernández, A., J. A. Yager, B. N. Wilkie, K. E. Leslie, and B. A. Mallard. 2005. Evaluation of bovine cutaneous delayed-type hypersensitivity (DTH) to various test antigens and a mitogen using several adjuvants. Vet. Immunol. Immunopathol. 104:45-58.

Hine, B. C., S. L. Cartwright, and B. A. Mallard. 2011. Effect of age and pregnancy status on adaptive immune responses of Canadian Holstein replacement heifers. J. Dairy Sci. 94:981-991.

Kelton, D. F., K. D. Lissemore, and R. E. Martin. 1998. Recommendations for recording and calculating the incidence of selected clinical diseases of dairy cattle. J. Dairy Sci. 81:2502-2509.

Kovac, M., E. Groeneveld, and L. A. Garcia-Cortes. 2002. VCE5, a package for the estimation of dispersion parameters. Proc. 7th World Congr. Genet. Appl. Livest. Prod., Montpellier, France.

LeBlanc, S. 2010. Monitoring metabolic health of dairy cattle in the transition period. J. Reprod. Dev. 56(Suppl.):S29-S35.

LeBlanc, S. J., K. E. Leslie, and T. F. Duffield. 2005. Metabolic predictors of displaced abomasum in dairy cattle. J. Dairy Sci. $88: 159-170$.

Mallard, B. A., H. Atalla, S. Cartwright, B. C. Hine, B. Hussey, M. Paibomesai, K. A. Thompson-Crispi, and L. Wagter-Lesperance. 2011. Genetic and epigenetic regulation of the bovine immune sys- 
tem: Practical implications of the high immune response technology. Pages 52-63 in Proc. National Mastitis Council 50th Annual Meeting. National Mastitis Council, Verona, WI.

Mallard, B. A., B. N. Wilkie, B. W. Kennedy, and M. Quinton. 1992. Use of estimated breeding values in a selection index to breed Yorkshire pigs for high and low immune and innate resistance factors. Anim. Biotechnol. 3:257-280.

Nino-Soto, M. I., A. Heriazon, M. Quinton, F. Miglior, K. Thompson, and B. A. Mallard. 2008. Differential gene expression of high and low immune responder Canadian Holstein dairy cows. Dev. Biol. (Basel) 132:315-320.

Sarker, N., M. Tsudzuki, M. Nishibori, H. Yasue, and Y. Yamamoto. 2000. Cell-mediated and humoral immunity and phagocytic ability in chicken lines divergently selected for serum immunoglobulin $\mathrm{M}$ and G levels. Poult. Sci. 79:1705-1709.

Sordillo, L. M., G. A. Contreras, and S. L. Aitken. 2009. Metabolic factors affecting the inflammatory response of periparturient dairy cows. Anim. Health Res. Rev. 10:53-63.

Ster, C., M. C. Loiselle, and P. Lacasse. 2012. Effect of postcalving serum nonesterified fatty acids concentration on the functionality of bovine immune cells. J. Dairy Sci. 95:708-717.
Thompson-Crispi, K. A., and B. A. Mallard. 2012. Type 1 and type 2 immune response profiles of commercial dairy cows in four regions of Canada. Can. J. Vet. Res. 76:120-128.

Thompson-Crispi, K. A., A. Sewalem, F. Miglior, and B. Mallard. 2012. Genetic parameters of adaptive immune response traits in Canadian Holsteins. J. Dairy Sci. 95:401-409.

Wagter, L., and B. A. Mallard, inventors. 2007. Method of identifying high immune response animals. University of Guelph, Guelph, ON, Canada, assignee. US Pat. No. 7,258,858.

Wagter, L. C., B. A. Mallard, B. N. Wilkie, K. E. Leslie, P. J. Boettcher, and J. C. Dekkers. 2000. A quantitative approach to classifying Holstein cows based on antibody responsiveness and its relationship to peripartum mastitis occurrence. J. Dairy Sci. 83:488-498.

Wilkie, B., and B. Mallard. 1999. Selection for high immune response: An alternative approach to animal health maintenance? Vet. Immunol. Immunopathol. 72:231-235.

Zhu, J., and W. E. Paul. 2008. $\mathrm{CD}_{4}$ T cells: Fates, functions, and faults. Blood 112:1557-1569. 